\title{
Index of seaweed algebras and integer partitions
}

\author{
Seunghyun Seo* \\ Department of Mathematics Education \\ Kangwon National University \\ Chuncheon, Republic of Korea \\ shyunseo@kangwon.ac.kr
}

\author{
Ae Ja Yee \\ Department of Mathematics \\ The Pennsylvania State University \\ University Park, PA, U.S.A. \\ auy20psu.edu
}

Submitted: Oct 10, 2019; Accepted: Feb 4, 2020; Published: Feb 21, 2020

(c) The authors. Released under the CC BY-ND license (International 4.0).

\begin{abstract}
The index of a Lie algebra is an important algebraic invariant. In 2000, Vladimir Dergachev and Alexandre Kirillov defined seaweed subalgebras of $\mathfrak{g l}_{n}\left(\right.$ or $\mathfrak{s l}_{n}$ ) and provided a formula for the index of a seaweed algebra using a certain graph, a so called meander.

In a recent paper, Vincent Coll, Andrew Mayers, and Nick Mayers defined a new statistic for partitions, namely the index of a partition, which arises from seaweed Lie algebras of type A. At the end of their paper, they presented an interesting conjecture, which involves integer partitions into odd parts. Motivated by their work, in this paper, we exploit various index statistics and the index weight generating functions for partitions. In particular, we examine their conjecture by considering the generating function for partitions into odd parts. We will also reprove another result from their paper using generating functions.
\end{abstract}

Mathematics Subject Classifications: 05A17, 11P81

\section{Introduction}

An integer partition $\lambda$ is a weakly decreasing finite sequence of positive integers $\lambda=$ $\left(\lambda_{1}, \lambda_{2}, \ldots, \lambda_{r}\right)[1]$. The $\lambda_{i}$ 's are called the parts of $\lambda$ and the sum of the parts is called the weight of $\lambda$. For a positive integer $n$, if the weight of $\lambda$ is $n$, then $\lambda$ is called a partition of $n$ and denoted by $\lambda \vdash n$. It is the convention that the empty sequence is the only partition of 0 .

In the theory of partitions, parity has played an important role. In [2], G. E. Andrews gave an extensive study on parity questions in partition identities. The very first example of Andrews mentioned in [2] is the following theorem by Euler.

\footnotetext{
*The first author was supported by Basic Science Research Program through the National Research Foundation of Korea(NRF) funded by the Ministry of Education(120180215).

†The second author was partially supported by a grant (\#633963) from the Simons Foundation.
} 
Euler's partition identity: The number of partitions of any positive integer $n$ into distinct parts equals the number of partitions of $n$ into odd parts.

Recently, the parity of a certain statistic assigned to partitions into odd parts was considered in the study of seaweed Lie algebras. In [4], V. Coll, A. Mayers, and N. Mayers defined a new statistic for partitions, namely the index of a partition, which arises from seaweed Lie algebras of type A. At the end of their paper, they presented a conjecture on the difference between the number of partitions of $n$ into odd parts with an odd index and the number of partitions of $n$ into odd parts with an even index. The definition of the index statistic requires some other concepts from Lie algebra, so we defer it to Section 2. Let $E_{\text {ind }}(n)$ denote the number of partitions of $n$ into odd parts with an odd index minus the number of partitions of $n$ into odd parts with an even index.

Conjecture 1 (Coll, Mayers, Mayers [4]).

$$
\sum_{n \geqslant 0}\left|E_{\text {ind }}(n)\right| q^{n}=\prod_{n=1}^{\infty} \frac{1}{1+(-1)^{n} q^{2 n-1}} .
$$

The main purpose of this paper is to study this counting function $E_{\text {ind }}(n)$. The following theorem is one of our main results.

Theorem 2. We have

$$
\sum_{n \geqslant 0}(-1)^{\left\lceil\frac{n}{2}\right\rceil} E_{\text {ind }}(n) q^{n}=\prod_{n=1}^{\infty} \frac{1}{1+(-1)^{n} q^{2 n-1}} .
$$

It follows from Theorem 2 that Conjecture 1 is equivalent to $(-1)^{\left\lceil\frac{n}{2}\right\rceil} E_{\text {ind }}(n) \geqslant 0$, i.e., the infinite product on the right hand side has nonnegative coefficients. The nonnegativity seems very difficult to prove, and their conjecture is still open.

Along with the index statistic arising in Conjecture 1, other index statistics can be defined on partitions. In particular, V. Coll, A. Mayers, and N. Mayers considered a case that is associated with partitions having parts of size one only, and they related that case to partitions into two-colored parts. In Section 5, we will treat this index statistic and exploit the generating functions for various subsets of partitions.

This paper is organized as follows. In Section 2, we will recollect some results on seaweed algebras from the literature. In Section 3, we give the definition of the index of a partition and prove Theorem 2 by considering a weight generating function for partitions. In Section 4, we will find the generating function weighted with another index statistic, which will reprove a result of V. Coll, A. Mayers, and N. Mayers. In Section 5, we will investigate further the index statistic considered in Section 4. We will then provide some remarks and further conjectures in the last section.

\section{Preliminaries}

A seaweed algebra is a subalgebra of $\mathfrak{s l}_{n}$, which was first introduced by V. Dergachev and A. Kirillov in [5]. The index of a Lie algebra is an important algebraic invariant in the 
study, which was given by J. Dixmier [6], and in the same paper, V. Dergachev and A. Kirillov provided a combinatorial algorithm to compute the index of a seaweed algebra using a certain graph, namely the meander of a seaweed algebra. Rather than giving the definitions of seaweed algebras and the index of Lie algebras, here we give a short description of the algorithm of V. Dergachev and A. Kirillov.

A seaweed algebra can be defined by two compositions $\lambda$ and $\mu$ of $n$. In this case, we say that the seaweed is of type $\frac{\lambda}{\mu}=\frac{\lambda_{1}|\cdots| \lambda_{r}}{\mu_{1}|\cdots| \mu_{s}}$.

The meander of a seaweed of type $\frac{\lambda_{1}|\cdots| \lambda_{r}}{\mu_{1}|\cdots| \mu_{s}}$ is a graph with $n$ vertices whose edges are given by $\lambda$ and $\mu$ as follows.

- Place the $n$ vertices in a row.

- First, partition the $n$ vertices into blocks of sizes $\lambda_{1}, \ldots, \lambda_{r}$, and let us call the vertices in the $i$-th block $v_{i, 1}, \ldots, v_{i, \lambda_{i}}$.

- Connect $v_{i, j}$ and $v_{i, \lambda_{i}+1-j}$ by an edge for $1 \leqslant j \leqslant\left\lfloor\lambda_{i} / 2\right\rfloor$. We draw these edges above the vertices and call them top edges.

- Repeat this process with $\mu$. This time, we draw edges below the vertices and call them bottom edges.

Figure 1 shows the meander of a seaweed of type $\frac{3|2| 1 \mid 1}{4 \mid 3}$.

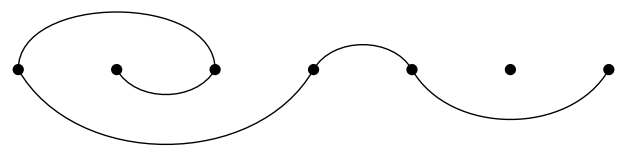

Figure 1: The meander of a seaweed of type $\frac{3|2| 1 \mid 1}{4 \mid 3}$

In [5], V. Dergachev and A. Kirillov showed that

$$
\operatorname{ind}_{\mu}(\lambda)=2 C+P-1,
$$

where $C$ and $P$ count the number of cycles and paths in the meander of a seaweed of type $\frac{\lambda}{\mu}$, respectively. Here, we regard a vertex of degree 0, i.e., an isolated vertex, as a path. In Figure 1, the index of the seaweed algebra is 1.

Note that in the meander associated with a seaweed of type $\frac{\lambda}{\mu}$, a vertex is connected to another vertex by a top edge (resp. a bottom edge) unless it is the middle point in a block of size equal to an odd part of $\lambda$ (resp. $\mu$ ). Since in a simple graph $G$, if a path is of length at least one, then each of its end vertices has degree 1, and an isolated vertex has degree 0 , it can be easily shown that in the meander associated to a seaweed of type $\frac{\lambda}{\mu}$,

$$
P=\frac{\mathrm{op}(\lambda)+\mathrm{op}(\mu)}{2}
$$

where op $(\lambda)$ count the number of odd parts in $\lambda$. Thus, by (1) and (2), we get

$$
\operatorname{ind}_{\mu}(\lambda) \equiv \frac{\mathrm{op}(\lambda)+\mathrm{op}(\mu)}{2}-1 \quad(\bmod 2) \text {. }
$$




\section{$3 \quad$ Parity difference of $\operatorname{ind}_{n}(\lambda)$}

We first define the index of a partition $\lambda$.

Definition 3. For a partition $\lambda$ of $n$, we define the index of $\lambda$ to be the index of the seaweed of type $\frac{\lambda}{n}$ and denote it by $\operatorname{ind}(\lambda)$, i.e.,

$$
\operatorname{ind}(\lambda):=\operatorname{ind}_{n}(\lambda)
$$

For $n=0$, if $\lambda$ is the empty partition, we define $\operatorname{ind}_{n}(\lambda)$ to be -1 .

In this section, we will consider the generating function for partitions with weight associated with index. Let $\mathcal{P}$ be the set of all partitions. For any subset $S$ of $\mathcal{P}$ and nonnegative integer $n$, define

$$
S_{n}:=\{\lambda \in S \mid \lambda \vdash n\} .
$$

Definition 4. Given a subset $S$ of $\mathcal{P}$, we define

$$
\begin{aligned}
& e_{S}(n):=\#\left\{\lambda \in S_{n} \mid \operatorname{ind}(\lambda) \text { is even }\right\}, \\
& o_{S}(n):=\#\left\{\lambda \in S_{n} \mid \operatorname{ind}(\lambda) \text { is odd }\right\} .
\end{aligned}
$$

We now consider the difference $o_{S}(n)-e_{S}(n)$.

Lemma 5. For any $n \geqslant 0$,

$$
\begin{aligned}
& o_{S}(n)-e_{S}(n) \\
& =\left\{\begin{array}{l}
\#\left\{\lambda \in S_{n} \mid \operatorname{op}(\lambda) \equiv 0(\bmod 4)\right\}-\#\left\{\lambda \in S_{n} \mid \operatorname{op}(\lambda) \equiv 2(\bmod 4)\right\} \quad \text { if } n \text { is even, } \\
\#\left\{\lambda \in S_{n} \mid \operatorname{op}(\lambda) \equiv 3(\bmod 4)\right\}-\#\left\{\lambda \in S_{n} \mid \operatorname{op}(\lambda) \equiv 1(\bmod 4)\right\} \quad \text { if } n \text { is odd. }
\end{array}\right.
\end{aligned}
$$

Proof. It easily follows from (3).

Definition 6. Given a subset $S$ of $\mathcal{P}$, let

$$
f_{S}(k, n):=\#\left\{\lambda \in S_{n} \mid \operatorname{op}(\lambda)=k\right\}
$$

and

$$
F_{S}(t, q):=\sum_{k, n \geqslant 0} f_{S}(k, n) t^{k} q^{n}
$$

From now on, we may omit the subscript $S$ in $o_{S}, e_{S}, f_{S}(k, n)$ and $F_{S}(t, q)$ if it is clear from the context.

Theorem 7. We have

$$
F(i,-i q)=\sum_{n \geqslant 0}(-1)^{\left\lceil\frac{n}{2}\right\rceil}(o(n)-e(n)) q^{n}
$$

where $i=\sqrt{-1}$. 
Proof. Note that for a partition $\lambda$ of $n$, if $n$ is even or odd, then $\operatorname{op}(\lambda)$ is even or odd, respectively. Thus,

$$
\begin{aligned}
F(t, q) & =\sum_{k, n: \text { even }} f(k, n) t^{k} q^{n}+\sum_{k, n: \text { odd }} f(k, n) t^{k} q^{n} \\
& =\sum_{k, n: \text { even }} f(k, n)\left(t^{2}\right)^{\frac{k}{2}} q^{n}+\sum_{k, n \text { :odd }} f(k, n) t^{-1}\left(t^{2}\right)^{\frac{k+1}{2}} q^{n}
\end{aligned}
$$

and we have

$$
\begin{aligned}
F(i,-i q) & =\sum_{k, n: \text { even }} f(k, n)(-1)^{\frac{k}{2}}(-1)^{\frac{n}{2}} q^{n}+\sum_{k, n: \text { odd }} f(k, n)(-1)^{\frac{k+1}{2}}(-1)^{\frac{n+1}{2}} q^{n} \\
& =\sum_{n: \text { even }}(-1)^{\frac{n}{2}}(o(n)-e(n)) q^{n}+\sum_{n: \text { odd }}(-1)^{\frac{n+1}{2}}(o(n)-e(n)) q^{n} \\
& =\sum_{n \geqslant 0}(-1)^{\left\lceil\frac{n}{2}\right\rceil}(o(n)-e(n)) q^{n},
\end{aligned}
$$

where the second equality follows from Lemma 5.

Throughout this paper, we adopt the following standard $q$-series notation:

$$
(a ; q)_{\infty}:=\lim _{n \rightarrow \infty} \prod_{j=0}^{n-1}\left(1-a q^{j}\right),
$$

and the following compressed notation:

$$
\left(a_{1}, \ldots, a_{m} ; q\right)_{\infty}:=\left(a_{1} ; q\right)_{\infty} \cdots\left(a_{m} ; q\right)_{\infty} .
$$

In what follows, we will see some special cases of Theorem 7 .

Corollary 8. We have

$$
\sum_{n \geqslant 0}(-1)^{\left\lceil\frac{n}{2}\right\rceil}\left(o_{\mathcal{P}}(n)-e_{\mathcal{P}}(n)\right) q^{n}=\frac{1}{\left(q,-q^{2} ;-q^{2}\right)_{\infty}} .
$$

Proof. Since $F_{\mathcal{P}}(t, q)=\frac{1}{\left(t q, q^{2} ; q^{2}\right)_{\infty}}$, we have $F_{\mathcal{P}}(i,-i q)=\frac{1}{\left(q,-q^{2} ;-q^{2}\right)_{\infty}}$.

Corollary 9. Let $\mathcal{D}:=\left\{\lambda \in \mathcal{P} \mid \lambda_{i}\right.$ 's are distinct $\}$. Then

$$
\sum_{n \geqslant 0}(-1)^{\left\lceil\frac{n}{2}\right\rceil}\left(o_{\mathcal{D}}(n)-e_{\mathcal{D}}(n)\right) q^{n}=\left(-q, q^{2} ;-q^{2}\right)_{\infty} .
$$

Proof. Since $F_{\mathcal{D}}(t, q)=\left(-t q,-q^{2} ; q^{2}\right)_{\infty}$, we have $F_{\mathcal{D}}(i,-i q)=\left(-q, q^{2} ;-q^{2}\right)_{\infty}$.

Corollary 10. For $d \geqslant 1$, let $\mathcal{O}^{d}:=\left\{\lambda \in \mathcal{P} \mid \lambda_{i}\right.$ 's are congruent to $\left.\pm 1(\bmod 4 d)\right\}$. Then

$$
\sum_{n \geqslant 0}(-1)^{\left\lceil\frac{n}{2}\right\rceil}\left(o_{\mathcal{O}^{d}}(n)-e_{\mathcal{O}^{d}}(n)\right) q^{n}=\frac{1}{\left(q,-q^{4 d-1} ; q^{4 d}\right)_{\infty}} .
$$


Proof. Since $F_{\mathcal{O}^{d}}(t, q)=\frac{1}{\left(t q, t q^{4 d-1} ; q^{4 d}\right)_{\infty}}, F_{\mathcal{O}^{d}}(i,-i q)=\frac{1}{\left(q,-q^{4 d-1} ; q^{4 d}\right)_{\infty}}$

Let $\mathcal{O}$ be the set of partitions into odd parts. Clearly, $\mathcal{O}=\mathcal{O}^{1}$, so $E_{\text {ind }}(n)=o_{\mathcal{O}}(n)-$ $e_{\mathcal{O}}(n)$. Therefore, Theorem 2 is a special case of Corollary 10.

\section{$4 \operatorname{ind}_{1^{n}}(\lambda)$}

In [4], V. Coll, A. Mayers, and N. Mayers also considered seaweed algebras of type $\frac{\lambda}{1^{|\lambda|}}$. For $k \geqslant 0$, let

$$
c_{n}(k):=\#\left\{\lambda \vdash n \mid \operatorname{ind}_{1^{n}}(\lambda)=n-k-1\right\}
$$

and

$$
c(k)=\lim _{n \rightarrow \infty} c_{n}(k) .
$$

Theorem 11 (Coll, Mayers, Mayers [4]). We have

$$
\sum_{k \geqslant 0} c(k) x^{k}=\frac{1}{(x ; x)_{\infty}^{2}} .
$$

They prove this theorem by constructing a bijection between the set of partitions counted by $c(k)$ and the set of two colored partitions. This result can also be proved by generating function manipulations.

First, note that in the meander associated with $\frac{\lambda}{1^{n}}$, there are no cycles. Thus, by (1) and (2), we get

$$
\operatorname{ind}_{1^{n}}(\lambda)=P-1=\frac{\mathrm{op}(\lambda)+n}{2}-1 .
$$

So, by the definition of $c_{n}(k)$, we see that

$$
c_{n}(k)=f_{\mathcal{P}}(n-2 k, n) .
$$

Let $f_{i}$ be the number of parts of size $i$ in $\lambda$. Then

$$
n=\sum_{i \geqslant 1} \lambda_{i}=\sum_{i \geqslant 1} i f_{i}=\sum_{i \geqslant 1} f_{2 i-1}+2 \sum_{i \geqslant 1} i\left(f_{2 i}+f_{2 i+1}\right) .
$$

Since $\lambda$ has $n-2 k$ odd parts, we get

$$
k=\sum_{i \geqslant 1} i\left(f_{2 i}+f_{2 i+1}\right) \geqslant \sum_{i \geqslant 1}\left(f_{2 i}+f_{2 i+1}\right),
$$

which shows that $\lambda$ can have at most $k$ parts of size greater than 1 and the largest part of $\lambda$ cannot exceed $2 k+1$. Thus, for a given $k, c_{n}(k)$ stabilizes as $n \rightarrow \infty$, and its limit $c(k)$ counts the number of partitions with parts greater than 1 such that $\sum_{i \geqslant 1} i\left(f_{2 i}+f_{2 i+1}\right)=k$, i.e.,

$$
c(k)=\#\left\{\lambda \mid f_{1}=0, \sum_{i \geqslant 1} i\left(f_{2 i}+f_{2 i+1}\right)=k\right\} .
$$


Define $\tilde{c}_{n}(k)$ by

$$
\tilde{c}_{n}(k)=\#\left\{\lambda \vdash n \mid f_{1}=0, \sum_{i \geqslant 1} i\left(f_{2 i}+f_{2 i+1}\right)=k\right\} .
$$

It is clear that $\sum_{n \geqslant 0} \tilde{c}_{n}(k)=c(k)$.

Let us consider the generating functions for $c_{n}(k)$ and $\tilde{c}_{n}(k)$. By $(5)$, we see that $c_{0}(0)=1$ and $c_{n}(k)=0$ if $n<2 k$.

Theorem 12. We have

$$
\begin{aligned}
\sum_{k, n \geqslant 0} c_{n}(k) t^{k} q^{n} & =\frac{1}{\left(q, t q^{2} ; t q^{2}\right)_{\infty}}, \\
\sum_{k, n \geqslant 0} \tilde{c}_{n}(k) t^{k} q^{n} & =\frac{1}{\left(t q^{2}, t q^{3} ; t q^{2}\right)_{\infty}} .
\end{aligned}
$$

Proof. Note that

$$
\begin{aligned}
\sum_{k, n \geqslant 0} c_{n}(k) t^{n-2 k} q^{n} & =\sum_{k, n \geqslant 0} f_{\mathcal{P}}(n-2 k, n) t^{n-2 k} q^{n} \\
& =F_{\mathcal{P}}(t, q) \\
& =\frac{1}{\left(t q, q^{2} ; q^{2}\right)_{\infty}} .
\end{aligned}
$$

By substituting $t^{-1 / 2}$ and $t^{1 / 2} q$ for $t$ and $q$, respectively, we get

$$
\sum_{k, n \geqslant 0} c_{n}(k) t^{k} q^{n}=F_{\mathcal{P}}\left(t^{-1 / 2}, t^{1 / 2} q\right)=\frac{1}{\left(q, t q^{2} ; t q^{2}\right)_{\infty}} .
$$

We now prove the second identity. Let

$$
\tilde{F}_{\mathcal{P}}(t, q)=\sum_{k, n \geqslant 0} \tilde{c}_{n}(k) t^{n-2 k} q^{n}
$$

By (6), we see that the partitions counted by $\tilde{c}_{n}(k)$ cannot have parts of size 1 . Thus

$$
\tilde{F}_{\mathcal{P}}(t, q)=(1-t q) F_{\mathcal{P}}(t, q) .
$$

Hence,

$$
\sum_{k, n \geqslant 0} \tilde{c}_{n}(k) t^{n-2 k} q^{n}=(1-t q) F_{\mathcal{P}}(t, q) .
$$

Upon substituting $t^{-1 / 2}$ and $t^{1 / 2} q$ for $t$ and $q$ above, we immediately get the second identity.

When $q=1$, the second identity of Theorem 12 yields Theorem 11 . 


\section{$5 \quad$ Parity difference of $\operatorname{ind}_{1^{n}}(\lambda)$}

We first define the conjugated index of a partition $\lambda$.

Definition 13. For a partition $\lambda$ of $n$, we define the conjugated index of $\lambda$ by the index of the seaweed of type $\frac{\lambda}{1^{n}}$ and denote it by $\operatorname{cind}(\lambda)$, i.e.,

$$
\operatorname{cind}(\lambda):=\operatorname{ind}_{1^{n}}(\lambda) .
$$

For $n=0$, if $\lambda$ is the empty partition, we define $\operatorname{ind}_{1^{n}}(\lambda)$ by -1 .

In this section, we will consider the generating function for partitions with the conjugated index weight.

Definition 14. Given a subset $S$ of $\mathcal{P}$, we define

$$
\begin{aligned}
& \bar{e}(n)=\bar{e}_{S}(n):=\#\left\{\lambda \in S_{n} \mid \operatorname{cind}(\lambda) \text { is even }\right\}, \\
& \bar{o}(n)=\bar{o}_{S}(n):=\#\left\{\lambda \in S_{n} \mid \operatorname{cind}(\lambda) \text { is odd }\right\} .
\end{aligned}
$$

We now consider the difference $\bar{o}(n)-\bar{e}(n)$.

Lemma 15. For any $n \geqslant 0$,

$$
\begin{aligned}
& (-1)^{\left\lfloor\frac{n}{2}\right\rfloor}(\bar{o}(n)-\bar{e}(n)) \\
& =\left\{\begin{array}{l}
\left.\#\left\{\lambda \in S_{n}: \operatorname{op}(\lambda) \equiv 0(\bmod 4)\right)\right\}-\#\left\{\lambda \in S_{n}: \operatorname{op}(\lambda) \equiv 2(\bmod 4)\right\} \quad \text { if } n \text { is even, } \\
\#\left\{\lambda \in S_{n}: \operatorname{op}(\lambda) \equiv 3(\bmod 4)\right\}-\#\left\{\lambda \in S_{n}: \operatorname{op}(\lambda) \equiv 1(\bmod 4)\right\} \quad \text { if } n \text { is odd. }
\end{array}\right.
\end{aligned}
$$

Proof. Note that $\mathrm{op}\left(1^{n}\right)=n$. Thus it easily follows from (3).

Recall $f(k, n)$ and $F(t, q)$ in Definition 6.

Theorem 16. We have

$$
F(i,-i q)=\sum_{n \geqslant 0}(-1)^{n}(\bar{o}(n)-\bar{e}(n)) q^{n},
$$

where $i=\sqrt{-1}$.

Proof. By (4), we see that

$$
\begin{aligned}
& F(i,-i q) \\
& =\sum_{k, n: \text { even }} f(k, n)(-1)^{\frac{k}{2}}(-1)^{\frac{n}{2}} q^{n}+\sum_{k, n: \text { odd }} f(k, n)(-1)^{\frac{k+1}{2}}(-1)^{\frac{n+1}{2}} q^{n} \\
& =\sum_{n: \text { even }}(-1)^{\frac{n}{2}}(\bar{o}(n)-\bar{e}(n))(-1)^{\frac{n}{2}} q^{n}+\sum_{n: \text { odd }}(-1)^{\frac{n-1}{2}}(\bar{o}(n)-\bar{e}(n))(-1)^{\frac{n+1}{2}} q^{n} \\
& =\sum_{n \geqslant 0}(-1)^{n}(\bar{o}(n)-\bar{e}(n)) q^{n}
\end{aligned}
$$

where the second equality follows from Lemma 15. 
By comparing Theorems 7 and 16, we get the following corollary.

Corollary 17. For any $n \geqslant 0$,

$$
o(n)-e(n)=(-1)^{\left\lfloor\frac{n}{2}\right\rfloor}(\bar{o}(n)-\bar{e}(n)) .
$$

In particular,

$$
|o(n)-e(n)|=|\bar{o}(n)-\bar{e}(n)| .
$$

Remark 18. Besides the partitions $\mu=n$ and $\mu=1^{n}$, we can consider other partitions of $n$.

1. Let $\mu$ be a partition of $n$ satisfying that

$$
\begin{cases}\operatorname{op}(\mu) \equiv 1(\bmod 4) & \text { if } n \text { is odd } \\ \operatorname{op}(\mu) \equiv 0(\bmod 4) & \text { if } n \text { is even. }\end{cases}
$$

For example, let $\mu=2^{\left\lfloor\frac{n}{2}\right\rfloor} 1^{n-2\left\lfloor\frac{n}{2}\right\rfloor}$. Then we have

$$
\operatorname{ind}_{\mu}(\lambda) \equiv \operatorname{ind}_{n}(\lambda) \quad(\bmod 2) .
$$

Thus $\#\left\{\lambda \in S_{n} \mid \operatorname{ind}_{\mu}(\lambda)\right.$ is odd (resp. even) $\}$ is the same as $o(n)$ (resp. $\left.e(n)\right)$. Moreover, the weight generating functions are the same.

2. Let $\mu$ be a partition of $n$ satisfying that

$$
\operatorname{op}(\mu) \equiv n \quad(\bmod 4)
$$

For example, let $\mu=4^{\left\lfloor\frac{n}{4}\right\rfloor} 1^{n-4\left\lfloor\frac{n}{4}\right\rfloor}$. Then we have

$$
\operatorname{ind}_{\mu}(\lambda) \equiv \operatorname{ind}_{1^{n}}(\lambda) \quad(\bmod 2) .
$$

Thus $\#\left\{\lambda \in S_{n} \mid \operatorname{ind}_{\mu}(\lambda)\right.$ is odd (resp. even) $\}$ is the same as $\bar{o}(n)$ (resp. $\left.\bar{e}(n)\right)$. Moreover, the weight generating functions are the same.

\section{Concluding Remarks}

In this section, we provide some remarks along with further conjectures related to index generating functions.

As mentioned in Introduction, Conjecture 1 is equivalent to the following conjecture.

Conjecture 19. For $n \geqslant 1$,

$$
(-1)^{\left\lceil\frac{n}{2}\right\rceil}\left(o_{\mathcal{O}}(n)-e_{\mathcal{O}}(n)\right)=\left|e_{\mathcal{O}}(n)-o_{\mathcal{O}}(n)\right| .
$$

It seems true that the non-negativity holds even if we take $\mathcal{O}^{d}$ for any $d \geqslant 1$. 
Conjecture 20. For $n \geqslant 0, d \geqslant 1$,

$$
(-1)^{\left\lceil\frac{n}{2}\right\rceil}\left(o_{\mathcal{O}^{d}}(n)-e_{\mathcal{O}^{d}}(n)\right)=\left|e_{\mathcal{O}^{d}}(n)-o_{\mathcal{O}^{d}}(n)\right| .
$$

Equivalently, for any $d \geqslant 1$,

$$
\frac{1}{\left(q,-q^{4 d-1} ; q^{4 d}\right)_{\infty}}
$$

has non-negative coefficients. In what follows, we list some conjectures that yields the non-negativity of the coefficients in (7).

Conjecture 21. For $m \geqslant 4$, every coefficient of

$$
\frac{1}{\left(q,-q^{m-1} ; q^{m}\right)_{\infty}}
$$

is nonnegative.

Conjecture 22 (increasing sequences by $\boldsymbol{m}$ ). For $m \geqslant 4$, there exists a positive integer $N(m)$ such that for any $n \geqslant N(m)$,

$$
\left[q^{n}\right] \frac{1}{\left(q,-q^{m-1} ; q^{m}\right)_{\infty}} \geqslant\left[q^{n-m}\right] \frac{1}{\left(q,-q^{m-1} ; q^{m}\right)_{\infty}},
$$

where $\left[q^{n}\right] F(q)$ denotes the coefficient of $q^{n}$ in the series $F(q)$.

Remark 23. Recently, Shane Chern proved that Conjecture 19 holds true for $n \geqslant 2.4 \times 10^{14}$ [3].

\section{Acknowledgements}

The authors would like to thank the anonymous referees for their careful reading and valuable comments.

\section{References}

[1] G. E. Andrews. The theory of partitions. Addison-Wesley, Reading, MA, 1976; reissued: Cambridge University Press, Cambridge, 1998.

[2] G. E. Andrews. Parity in partition identities. Ramanujan J., 23 (1-3): 45-90, (2010).

[3] S. Chern. Nonmodular infinite products and a Conjecture of Seo and Yee. arXiv: 1912.10341, 2019.

[4] V. Coll, A. Mayers, and N. Mayers. Statistics on partitions arising from seaweed algebras. arXiv:1809.09271, 2018.

[5] V. Dergachev and A. Kirillov. Index of Lie algebra of seaweed type J. Lie Theory, 10 (2): 331-343 (2000).

[6] J. Dixmier. Enveloping algebras. North-Holland Mathematical Library, Vol. 14. Translated from the French. North-Holland Publishing Co., Amsterdam-New YorkOxford, 1977. 